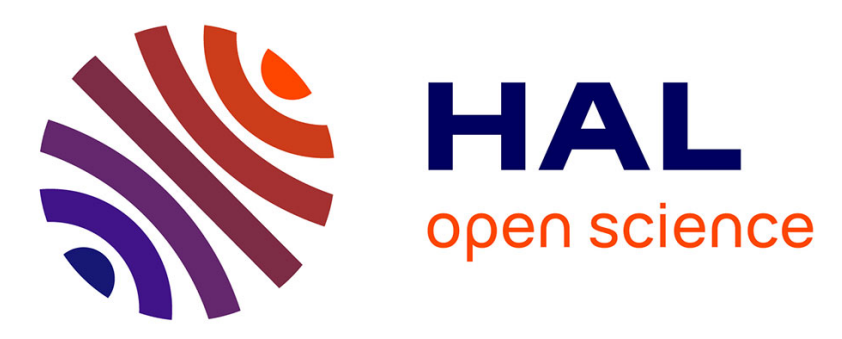

\title{
Numerical and experimental investigation of an underplatform damper test rig
}

Luca Pesaresi, Loic Salles, Bob Elliott, Adrian Jones, Jeffrey S. Green, Christoph W. Schwingshackl

\section{- To cite this version:}

Luca Pesaresi, Loic Salles, Bob Elliott, Adrian Jones, Jeffrey S. Green, et al.. Numerical and experimental investigation of an underplatform damper test rig. Applied Mechanics and Materials, 2016, 849, pp.1-12. 10.4028/www.scientific.net/AMM.849.1 . hal-02543859

\section{HAL Id: hal-02543859 \\ https://hal.science/hal-02543859}

Submitted on 15 Apr 2020

HAL is a multi-disciplinary open access archive for the deposit and dissemination of scientific research documents, whether they are published or not. The documents may come from teaching and research institutions in France or abroad, or from public or private research centers.
L'archive ouverte pluridisciplinaire HAL, est destinée au dépôt et à la diffusion de documents scientifiques de niveau recherche, publiés ou non, émanant des établissements d'enseignement et de recherche français ou étrangers, des laboratoires publics ou privés. 


\title{
Numerical and experimental investigation of an underplatform damper test rig
}

\author{
L.Pesaresi $^{1, \mathrm{a}}{ }$ L.Salles $^{1}$, R.Elliott ${ }^{2}$, A.Jones $^{2}$, J.S.Green ${ }^{2}$, C.W.Schwingshackl ${ }^{1}$ \\ ${ }^{1}$ Imperial College London, London SW7 2AZ, UK \\ ${ }^{2}$ Rolls-Royce plc, Derby DE24 8BJ, UK \\ aluca.pesaresi12@imperial.ac.uk
}

Keywords: Turbine blade vibrations, Nonlinear dynamics, Friction damping, Underplatform dampers, Experimental test rig

\begin{abstract}
During operation mechanical structures can experience large vibration amplitudes. One of the challenges encountered in gas-turbine blade design is avoiding high-cycle fatigue failure usually caused by large resonance stresses driven by aeroelastic excitation. A common approach to control the amplitude levels relies on increasing friction damping by incorporating underplatform dampers (UPD). An accurate prediction of the dynamics of a blade-damper system is quite challenging, due to the highly nonlinear nature of the friction interfaces and detailed validation is required to ensure that a good modelling approach is selected. To support the validation process, a newly developed experimental damper rig will be presented, based on a set of newly introduced non-dimensional parameters that ensure a similar dynamic behaviour of the test rig to a real turbine blade-damper system. An initial experimental investigation highlighted the sensitivity of the measured response with regards to settling and running in of the damper, and further measurements identified a strong dependence of the nonlinear behaviour to localised damper motion. Numerical simulations of the damper rig with a simple macroslip damper model were performed during the preliminary design, and a comparison to the measured data highlighted the ability of the basic implicit model to capture the resonance frequencies of the system accurately.
\end{abstract}

\section{Introduction}

One of the main problems encountered in turbine blade design is avoiding high-cycle fatigue caused by large resonance stresses [1]. Different approaches for lowering the amplitude levels and the stresses in a turbine are available, one of the most common relying on energy dissipation at dry friction contacts of the blade. This energy dissipation can reduce the vibration amplitude significantly, but it also leads to shifts in the resonance frequencies of the bladed disk. Dry friction can occur in various locations in a blade (root, shrouds etc.), however the most effective practical solution is an underplatform damper [2]. The underplatform damper consists of a metal device which sits in a groove on the underside of the platform between adjacent blades, and it is kept in place and loaded by the centrifugal force (see Fig. 1). When the blades vibrate, the relative motion between the blades and the damper leads to friction in the contact interface, which in turn provides energy dissipation and damping to the system.

The dynamics of a bladed disk assembly, with underplatform dampers, is governed by nonlinear differential equations [3-5]. Many studies on damper modelling have been conducted in the past [6-13], but despite all the research efforts, there is still not a standardised approach for the analysis. For this study initially, the blade was modelled as a single degree of freedom system [7,14], followed by multi-degrees of freedom models with some kinematic hypothesis on the damper motion (eg. parallel platform and damper surfaces during motion) $[8,10,15]$. More recently detailed full 3D models were introduced to more accurately capture the damper motion [11]. The modelling approaches developed must be validated against experimental data to ensure the correct prediction of the nonlinear behaviour. The most common experimental set-up is a double beam with platforms and a damper fitted in between with centrifugal load provided via wires $[10,16,17]$. A complete rotating blisk with 
cottage-roof dampers has also been tested [18] allowing full engine order excitation. A full not rotating blisk has recently been developed by Berruti et al. $[19,20]$ overcoming the lack of symmetry in the double beam set up. Test rigs based on blisks can better mimic the effect of dampers on a real turbine stage, but the inevitable mistuning introduced due to the manufacturing and the asymmetries in the loading of the dampers makes the analysis more complicated. Most of these rigs focused on capturing the nonlinear effect correctly, but did not consider a realistic blade-platform motion in great detail.

To overcome these difficulties a new experimental underplatform damper rig is presented in this paper, which follows the scheme of previously developed rigs $[10,16]$ with two pseudo-blades and one damper. However the geometry has been tuned to allow a more realistic damper motion and a similar friction damping on the blade dynamics. For this purpose, a set of non-dimensional parameters partly new and partly selected from literature, has been used. Nonlinear simulations with an implicit damper model [21] have been performed to support the design and a study on their abilities to capture the experimental FRFs of the introduced test rig will be presented.

\section{UPD rig development}

Rig concept. The underplatform damper (UPD) test rig is an experimental set up that allows the effect of UPDs on blade like structures to be evaluated. A static rig design was chosen, to isolate the effect of the damper on the dynamics from the mistuning effect and to create a simplified experimental set up. The basic concept of the rig can be seen in Fig. 1. Two pseudo beam like blades are fixed on a common base, which simulates a rigid disk. The damper is a wedge type, [10], which has a triangular cross section with a characteristic angle. Unlike a real high pressure turbine blade (HPT), the aerofoil is substituted by a straight rectangular cross-section beam, given the similar vibration modes of the beam like blade to a real blade. These simplifications may lead to a different nonlinear dynamic behaviour, and for this reason, it is important to tune the geometry to minimise this difference.

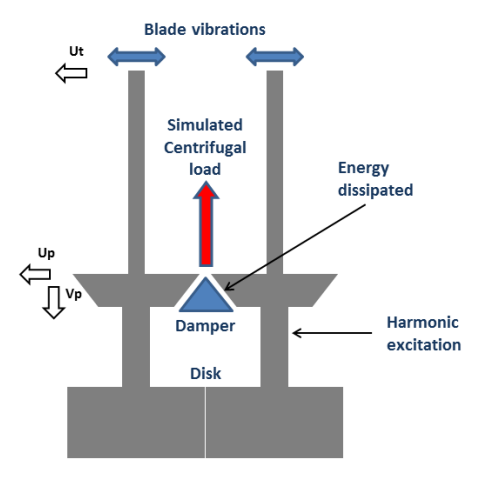

Fig. 1: Basic concept of the UPD rig

Non-dimensional parameters. In order to make the design of the test rig as realistic as possible, it is necessary to tune the geometry to reflect the dynamics of real blades. For this purpose a set of nondimensional design parameters (Table 1) characterising the main aspects of the dynamic behaviour of a turbine blade has been introduced. These parameters, in part selected from the literature $[10,14,22]$ and in part newly defined, are:

A) The force ratio [14] of the centrifugal load over the excitation force controls the regime in which the damper is operating. At a low ratio, the excitation force is predominant and the damper is in "slip" condition, whereas at very high levels the damper is in full stuck condition. During the measurement this parameter can be varied by modifying the damper pull load or the shaker force. 
Table 1: Non-dimensional parameters.

\begin{tabular}{lcc}
\hline & Description & Expression \\
\hline A) & Force ratio & $\frac{C F}{F_{\text {exc }}}$ \\
B) & Acceleration ratio & $\frac{r \Omega^{2}}{a \omega^{2}}$ \\
C) & Displacement ratio & $\frac{u_{p}}{u_{t}}$ \\
D) & Platform displacement ratio & $\frac{u_{p}}{v_{p}}$ \\
E) & Frequency shift & $\frac{\omega_{d}}{\omega_{u}}$ \\
F) & Energy ratio & $\frac{E_{f r i}}{E_{t o t}}$ \\
G) & Contact strain & $\frac{\sigma}{E}$
\end{tabular}

B) The acceleration ratio, in a real engine, represents the ratio between the centrifugal acceleration of the damper, due to the rotational speed, and the acceleration of the blade platform projected along the same direction. This parameter controls whether the damper separates from the platform when the damper acceleration is exceeded by the platform acceleration. In the simplified test rig the damper maximum acceleration is governed by the flexibility of the wire-pulley system.

C) The displacement ratio [22] is the ratio between the horizontal displacement of the platform and the horizontal displacement of the blade tip in the first flexural mode of the blade. Higher values cause more relative motion at the damper interface leading to more energy dissipated. It allows similar mode shapes between the real blade and the test rig.

D) The platform displacement ratio is the ratio between the horizontal and vertical displacement of the platform. This parameter influences the kinematics of the damper during a vibration cycle and ensures realistic damper motions.

E) The frequency shift [10] indicates the change in frequency caused by the damper compared to the baseline configuration (no damper). The damper causes a stiffening of the system due to the coupling of the blades which depends on the load, on the mode considered, and on the platform and damper geometry. It will ensure that the chosen design leads to realistic frequency shifts.

F) The energy ratio considers the energy dissipated by the friction damping and the energy dissipated due to the material hysteresis. It quantifies the contribution of the damper to the total energy dissipation in the dynamic system.

G) The contact strain is the ratio between the contact pressure and the Young's modulus of the material used at the interface. Keeping it similar to a real blade allows the rig to reproduce a similar pressure distribution and similar wear behaviour. 
Design features and measurement set-up. The design process of the UPD test rig was driven by the above mentioned parameters and by minimising external factors that would affect the quality of the nonlinear measurements. The geometry of the blade was defined and optimised using linear FE and nonlinear dynamic analysis, leading to the final design shown in Fig. 2. In particular, the beam length relative to the platform height was adjusted in order to match the displacement ratio and frequency shift of a real blade. In addition, the platform displacement ratio was directly influenced by changing the platform width. The correct contact strain at the blade-platform interface was reached by adjusting the damper dimensions. The centrifugal force, which the damper experiences in a real engine, is simulated with a pulley system and a set of variable weights. In order to have a correct acceleration ratio (defined above) to control damper separation and clapping, the stiffness $\mathrm{K}$ of the stainless steel pulling wire is chosen to satisfy the following relation:

$$
K_{w}=\frac{E_{w} A}{l}>m \omega^{2}
$$

where $K_{w}$ is the stiffness of the wire, $E_{w}$ is the equivalent Young's modulus of the wire, $\mathrm{A}$ is the nominal cross section area of the wire, 1 is the free length between the damper and the first pulley, $\mathrm{m}$ is the mass of the damper and $\omega$ is the vibration frequency of the blades. It must be noted that the equivalent Young's modulus E is not constant and it increases with the load, since the strands of the wire tend to get closer. The energy parameter, newly introduced here, is affected by all the other parameters, and reasonable values can be achieved only after some design iterations. Compared to a real HPT blade, the fir-tree attachment used to connect the blade to the disk is removed in order to minimise the number of friction joints and focus the non linear rig dynamics on the damper effects alone. The correct alignment between two consecutive platforms, which guarantees a symmetrical loading of the damper, is ensured by a shim between the two blocks (see Fig. 2). The test rig was manufactured to the defined design and the blades were then clamped tightly to a 2 tons inertia block via a newly developed hydraulic vice capable of $200 \mathrm{kN}$ clamping force. The measurement set up can be seen in Fig. 2. The excitation is provided by an electrodynamic shaker, attached below the platform, and the response is measured with two non-contact laser doppler vibrometers (LDV) focused near the tip of each blade.
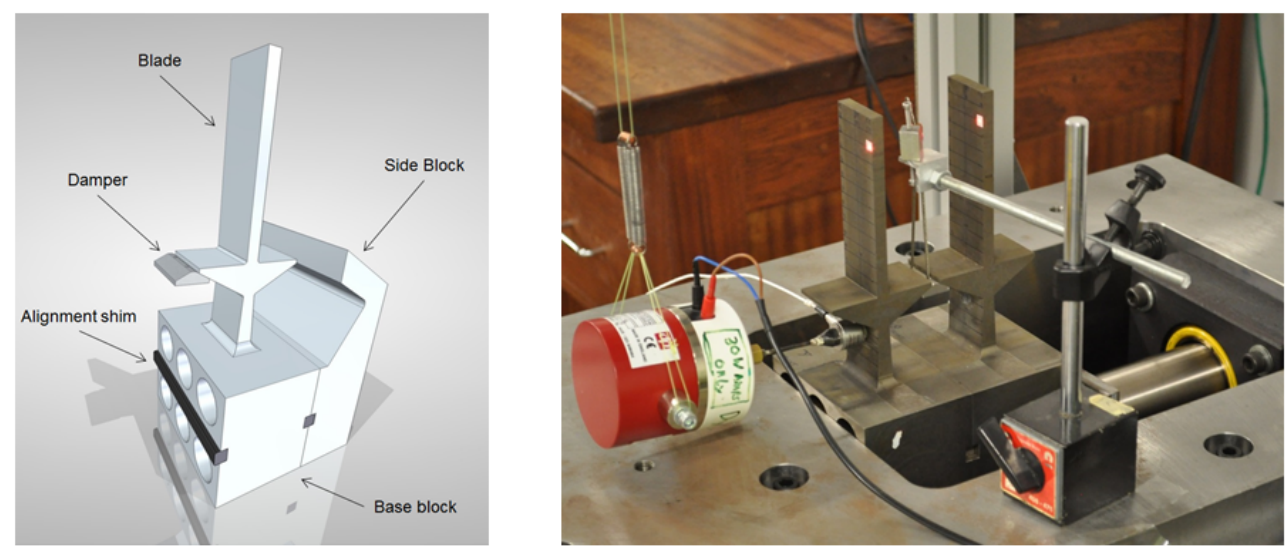

Fig. 2: Rig design and lab set-up CRolls-Royce plc. 


\section{Measurements}

Rig set-up assessment. When evaluating the non linear dynamic response caused by friction damping, it is very important that the underlying dynamic system without a damper is linear and the nonlinearities introduced by the clamping are minimal [23,24]. For this reason, frequency sweeps of the rig without the damper at increasing clamping forces have been performed, and the modal parameters were extracted (Fig. 3a) to obtain an understanding of the damping performance.

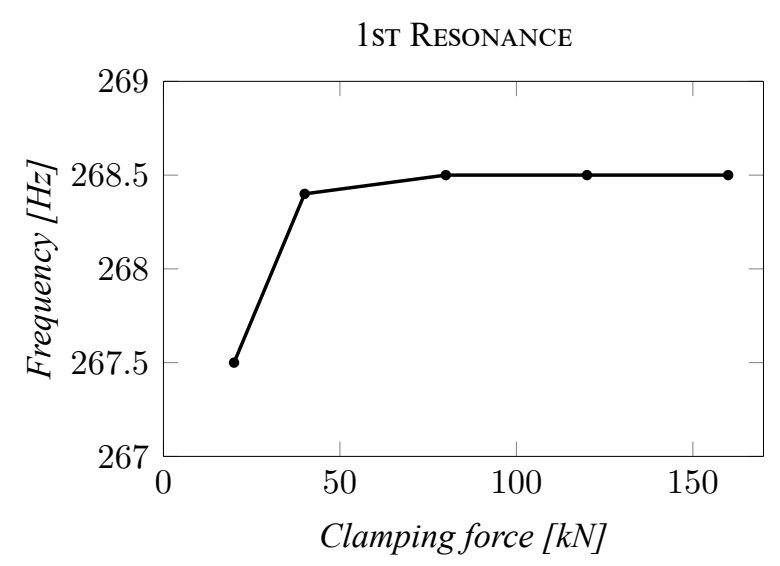

(a)

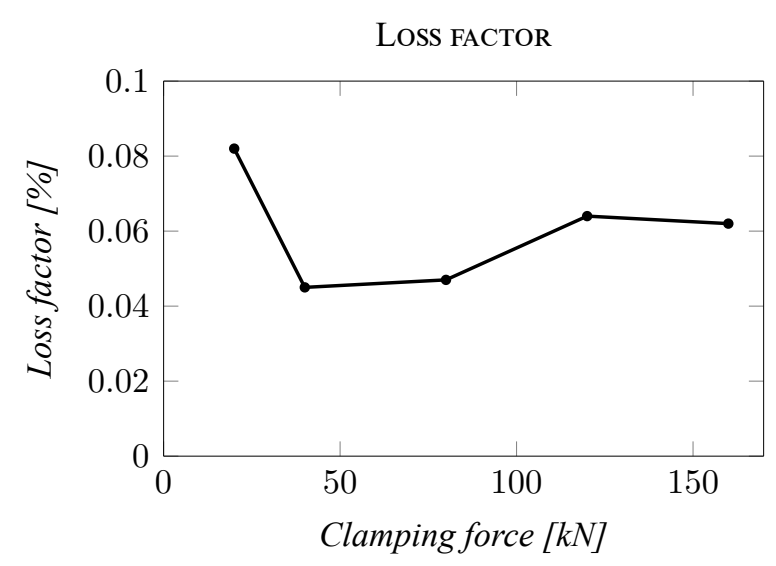

(b)

Fig. 3: Clamping effect on the 1F modal parameters a) Resonance frequency b) Loss factor

Both loss factor and resonance frequency show some small variations at low clamping forces but seem to converge above $30 \mathrm{kN}$. The small influence of the clamp on the dynamics is confirmed by the values of the loss factor which are below $0.1 \%$, which is very close to the free-free configuration of the blade. In order to evaluate the nonlinear behaviour of the rig at a given force ratio, which should guarantee a constant response as suggested in the literature [7, 14,17], various FRFs have been measured over a wide range of damper loads, each time adjusting the harmonic excitation to maintain the same force ratio. In this condition, the FRFs should be similar, since similar levels of nonlinearities are triggered, however, our findings (Fig. 4) show a clear change in the resonance frequency at low damper load. A possible explanation could be an inaccurate settling of the damper, leading to a non uniform contact at the interface. When the damper load exceeds $700 \mathrm{~N}$ the resonance frequency of the first mode stabilises, providing a minimum loading condition for the subsequent tests.

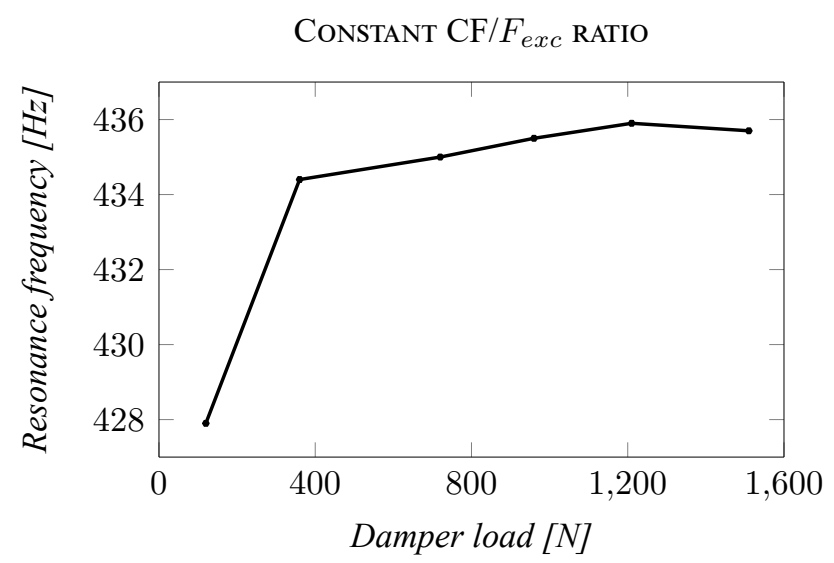

Fig. 4: FRFs at constant force ratio and variable damper load 
Non-linear measurements. The initial nonlinear measurements focussed on the first flexural mode $(1 \mathrm{~F})$, as it normally leads to the highest alternating stresses near the blade root. The double blade configuration of the rig leads to an in phase (IP) and out of phase (OoP) $1 \mathrm{~F}$ mode, whereas in a real engine, the inter blade phase angle (phase between two consecutive blades) will be between these two extreme values depending on the nodal diameter excited. The pulling load for the damper was kept at a high level $(960 \mathrm{~N})$ to ensure a good conformity at the contact interface. A stepped sine test with a Data Physics SignalCalc ACE card was performed around each resonance at various levels of excitation, from $0.01 \mathrm{~N}$ to $17 \mathrm{~N}$. A feedback control kept the excitation force constant throughout the sweep, which guarantees that the nonlinear behaviour is activated every time in a similar way.

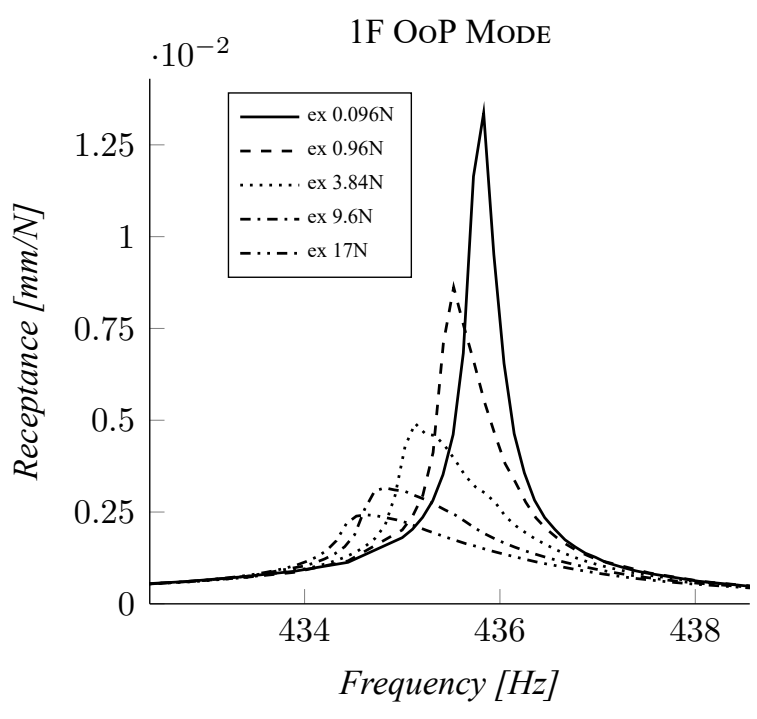

(a)

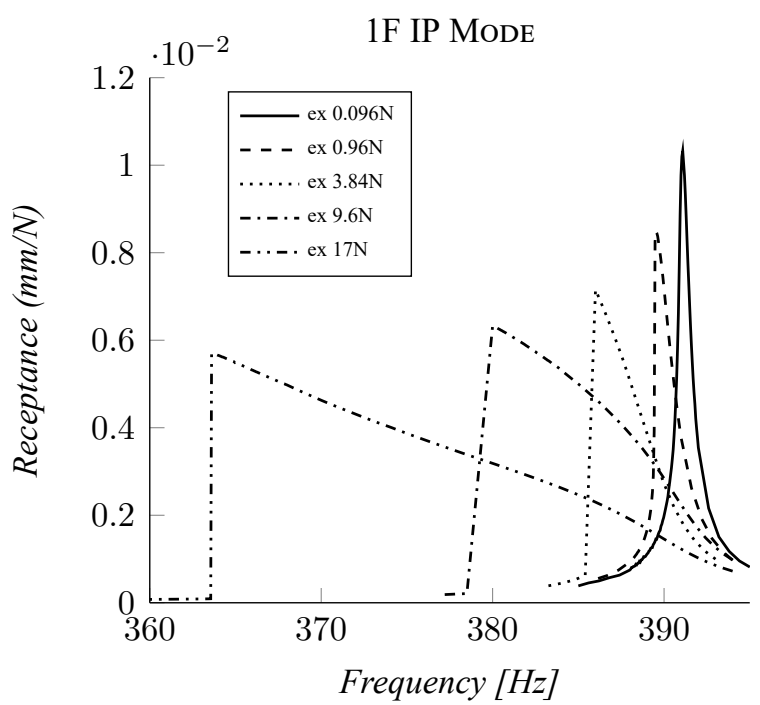

(b)

Fig. 5: Experimental FRFs at various excitation forces and constant damper load (960 N)

The out of phase mode (OOP) in Fig. 5a shows a significant amplitude reduction from the nearly linear case $\left(C F / F_{e x c}=10000\right)$ increasing the excitation force. This could be explained by the strong contribution of the local microslip on the energy dissipation mechanism as, even at low excitation levels, when the macroslip is not triggered, the damper is still effective. A very slight frequency shift can also be observed (less than 1\%) from the linear to the more damped measurements.

The in phase mode (Fig. 5b), shows a slightly lower amplitude reduction, and in addition large jumps appear at low force ratios due to the strong nonlinear softening in the system. Different maxima are reached when the sweep direction is changed, as the position of the jump, on the two stable FRF branches, shifts. The strong softening effect observed in the in phase FRFs (see Fig. 5b) has a significant impact on the blade dynamics, as it causes large shifts in the resonance frequency compared to the linear case. A possible explanation could be based on the tendency of the damper to roll in the IP mode $[10,17]$, which can reduce the damper-platform contact area at higher amplitudes, leading to a softer system. In particular, the contact on the platform which is lifting tends to shift down close to the lower edge and the contact on the platform which is lowering tends to move up close to the upper edge.

To confirm the previous assumption on the damper motion, a local measurement of the damper kinematics has been performed for the IP and OOP modes. Two LDV single-point lasers were directed by two mirrors to the bottom corners of the damper as shown in Fig. 6a similarly to the set up shown in [17]. It can be seen that for the IP mode the corners of the damper are moving out of phase, causing a rolling motion, whereas the OOP mode shows a pure translation of the damper (Fig. 6b). This confirms the strong difference in damper motion between the IP and OOP mode, and highlights the significance on the overall blade behaviour. 


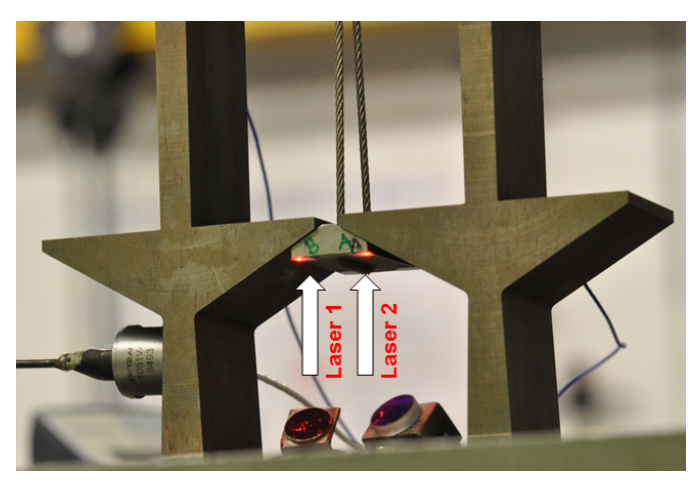

(a)
1F OoP BLADES
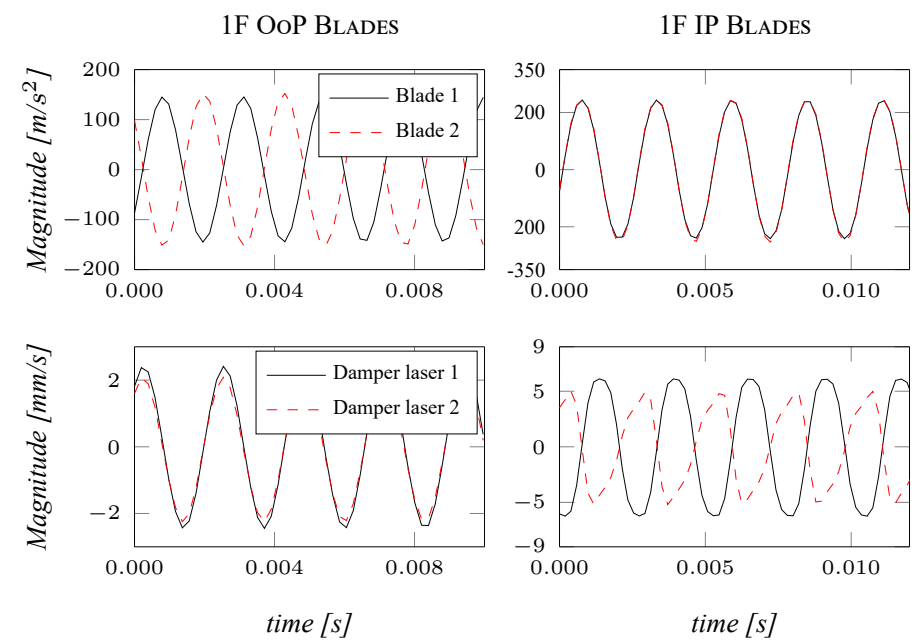

(b)

Fig. 6: a) Damper kinematics measurement position, b) Damper motion for the IP and OoP blades modes

\section{Numerical simulations}

Model. A model of the rig was generated in order to help with the understanding of the damper behaviour and identify possible uncertainties with the rig design. The analysis, conducted in FORSE (Forced Response SuitE), is based on a multi-harmonic balance solver with a powerful model reduction technique [25-27]. To allow a fast computation, an implicit model of the wedge damper [21] was used in this investigation, which consists of a lumped mass at the centre of gravity of the damper, coupled to the blade platforms by the macroslip friction elements as shown in Fig. 7.

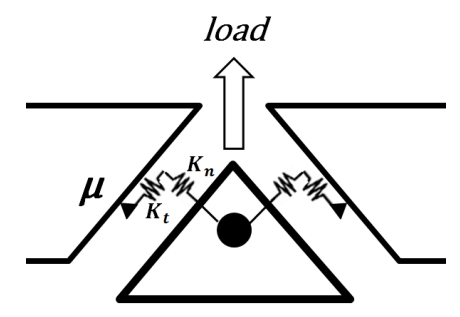

Fig. 7: Cottage-roof damper model

Normal load variation, including separation of the contact interface is considered in the model, as well as the tangential friction forces, responsible for the stick-slip transitions. The interface parameters required for the contact model are the friction coefficient $\mu$, and the normal and tangential contact stiffness $\mathrm{kn}$ and $\mathrm{kt}$. No limiting hypothesis is made on the damper motion, which is calculated instead iteratively as a result of the coupled blade-damper dynamic problem. The element allows for 3D translational motion, but no rotating DOFs are considered.

For the analysis, a detailed FE model of the UPD rig was created, and updated to accurately represent the dynamics of the linear rig without a damper. Great care was taken to identify the correct interface parameters for the nonlinear element, as they can significantly influence the results [28]. Measured values for the tangential and normal contact stiffness and the friction coefficient $(\mu=0.6)$ were used to ensure a realistic model $[29,30]$. The UPD element is connected to a central node at the contact interface of the linear FE model and the contact area of each damper side must be specified. 
The initial static normal load $N_{1}$ and $N_{2}$ for each side of the damper is calculated by taking friction into account [21]:

$$
N_{1}=N_{2}=\frac{1}{2} \frac{F}{(\cos (\alpha)+\mu \sin (\alpha))}
$$

where $F$ is the radial force due to the CF loading and $\alpha$ is the damper angle.

For the multiharmonic analysis, the first three harmonics were included in the Fourier series, as it has been shown that this allowed the most significant nonlinear phenomena to be accurately captured [28] in most cases. Modal damping extracted from a single blade test was used as baseline material damping $(\zeta=0.05 \%)$. The position of the sinusoidal excitation and the location of the output response was chosen to coincide with the experiment (see Fig. 2).

Results. The plot in Fig. 8a shows a numerical FRF for the IP (left peak) and OOP (right peak) first flexural mode at the lowest excitation level ( force ratio $=10000$ ). At this level, both friction contact elements at the two sides of the damper are stuck, and therefore, no energy dissipation occurs. The magnitude of the OOP mode is close to the measurements in the linear case, however the IP mode results in much higher amplitudes, indicating a possible dissipation in the test rig even at very low amplitudes, which is not captured correctly with the implicit damper model. A possible explanation for this could be the detected rolling motion of the damper in the IP mode, which can not be described by the basic UPD model. The shifted resonance frequencies are close to the measured ones, with the IP mode about $3 \%$ higher, and the OOP about $5 \%$ lower, which highlights the capability of the element to introduce the correct stiffness between the two blades.

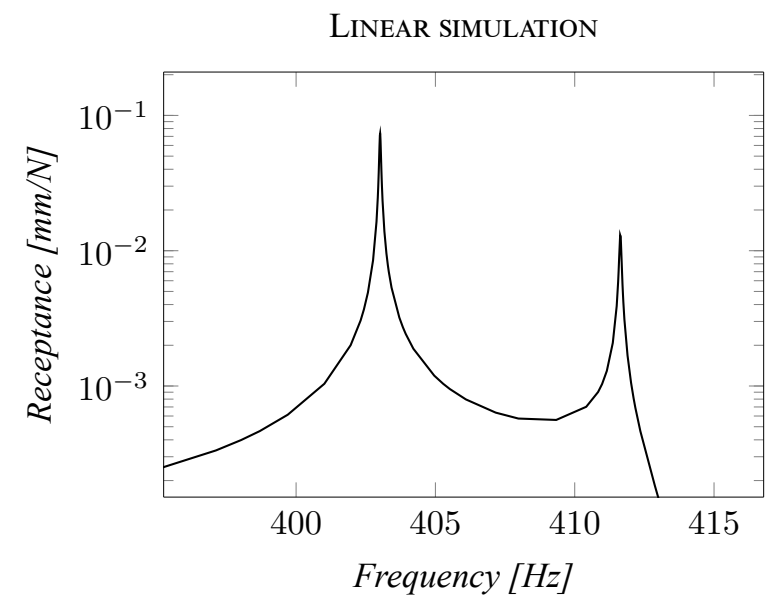

(a)

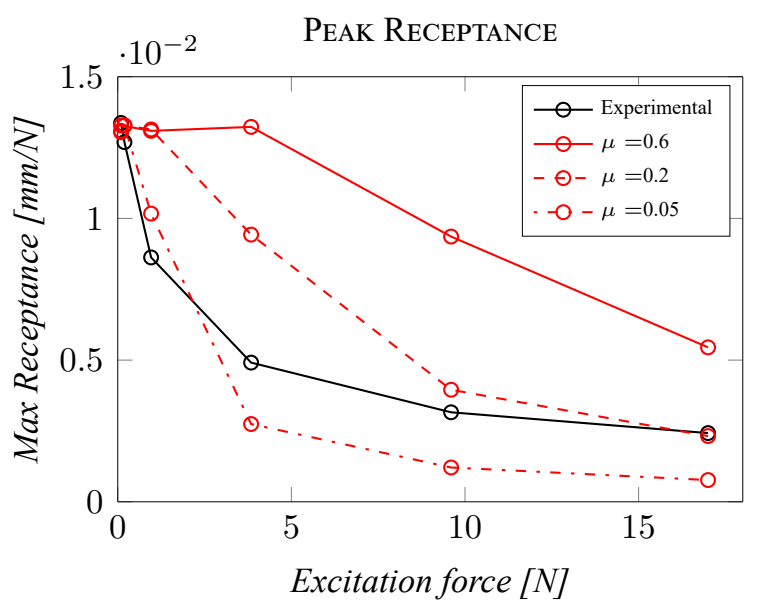

(b)

Fig. 8: a) IP (left) and OoP (right) linear simulation, b) OoP Numerical NL simulation and experiments

The nonlinear analysis was focused on the OOP mode due to the difficulty of this modelling approach to capture the IP mode correctly. The amplitude reduction caused by the friction dampers was one of the main design concerns during the initial investigation, and it can be used to evaluate the accuracy of the modelling approach. Fig. $8 \mathrm{~b}$ shows the peak magnitude of the receptance for the OOP mode at different excitation levels, both experimental and numerical. In the experimental curves, a drop in peak amplitude can be observed, even at very low force levels, indicating unexpected energy dissipation. The model, based on the measured contact interface parameters, is unable to correctly capture this initial drop, but it tends to get closer to the experimental amplitudes at higher excitation levels. This difference may indicate that microslip phenomena are taking place at the contact interface, which can not be captured with the basic macroslip model, leading to incorrect amplitude predictions at very low excitation levels. To better capture the contribution of the microslip with a macroslip model, the 
friction contact parameters were adjusted to achieve a better match at lower amplitudes as suggested in [31]. With a friction coefficient of $\mu=0.2$, much better results were obtained at high excitation levels (see Fig. 8b), but still some discrepancies were observed for lower excitation levels. A further decrease in the friction coefficient to a very low value of $\mu=0.05$ helped to get a good match in the low excitation zone, however it overestimates the damping effect at higher forcing levels. For these reasons, a simple macroslip UPD element is a very helpful tool during preliminary design stages to capture the shifts in frequencies in the model, but care needs to be taken in the prediction of the resulting amplitudes, due to its intrinsic inability to correctly capture microslip phenomena. Tuning the friction coefficient could improve the results, however it is hard to find an optimal value for a wide range of excitation forces. In addition, the IP mode dynamics, which seems to be strongly influenced by the rotation of the damper, can not be captured correctly by this simple implicit damper model.

\section{Conclusions}

A new static underplatform damper test rig, made of two pseudo blades has been designed and commissioned. A set of non-dimensional parameters has been suggested to ensure a dynamic behaviour of the test rig with realistic loading, displacement and stiffness characteristics. During commissioning of the rig, the settling of the damper was identified as the main issue affecting the quality of the measurements. Nonlinear measurements were performed for the first bending IP and OOP mode at a range of forcing levels, to explore the dynamic behaviour of the rig. The OOP mode showed significant nonlinear damping, whereas the IP mode showed a marked softening effect, which reflects the influence of the rotation of the damper.

A simple UPD model with two macroslip friction elements was used for the analysis of the real damper. This approach allowed a fast computation during the preliminary design phase and a comparison with the final rig behaviour demonstrated a good representation of the frequency shifts caused by the damper. However, the macroslip nature of the UPD element did not allow small excitation amplitudes to be captured accurately, due to an expected presence of microslip in the rig. Using the friction coefficient as a tuning parameter led to a slightly better damping prediction, but relatively low values were required, which may not have a physical meaning for a dry friction interface. Due to the inability of the model to capture the rotation of the damper and its effect on the dynamics, particular care needs to be taken when evaluating lower nodal diameters of the bladed disks where the dynamic behaviour of adjacent blades approaches an IP mode. When more accurate predictions are required over a wide range of force levels, some more advanced modelling approaches that can capture the microslip phenomena and rotational effects are required.

\section{ACKNOWLEDGEMENTS}

The authors are grateful to Rolls-Royce Plc. and Innovate UK for providing the financial support for this project and for giving permission to publish this work. 


\section{References}

[1] B.A.Cowles, "High cycle fatigue in aircraft gas turbine - an industry prospective," International Journal of Fracture, vol. 80, pp. 147-163, 1996.

[2] J. H. Griffin, “A review of friction damping of turbine blade vibration," International Journal of Turbo and Jet Engines, vol. 7, pp. 297-307, 1990.

[3] B. Feeny, N. Hinrichs, and K. Popp, "A historical review on dry friction and stick-slip phenomena," Applied Mechanics Reviews, vol. 51, no. 5, pp. 321-341, 1998.

[4] L. Gaul and R. Nitsche, "The role of friction In mechanical joints," Applied Mechanics Reviews, 2001.

[5] J. P. Den Hartog, "Forced vibrations with combined coulomb and viscous friction," ASME trans., pp. 107-115, 1931.

[6] G. C. K. Yeh, "Forced vibrations of a two degree of freedom system with combined coulomb and viscous damping," The Journal of the Acoustical Society of America, vol. 39, no. 1, 1966.

[7] J. H. Griffin, "Friction Damping of Resonant Stresses in Gas Turbine Engine Airfoils," Journal of Engineering for Power, vol. 102, pp. 329-333, 1980.

[8] B. D. Yang and C. H. Menq, "Characterization of Contact Kinematics and Application to the Design of Wedge Dampers in Turbomachinery Blading : Part 1 - Stick-Slip Contact Kinematics," Journal of Engineering for Gas Turbines and Power, vol. 120, no. April 1998, pp. 410-417, 1998.

[9] J. H. Wang and W. K. Chen, "Investigation of the Vibration of a Blade With Friction Damper by HBM," Journal of Engineering for Gas Turbines and Power, vol. 115, no. April 1993, pp. 294299, 1993.

[10] K. Y. Sanliturk, D. J. Ewins, and A. B. Stanbridge, "Underplatform Dampers for Turbine Blades: Theoretical Modeling, Analysis, and Comparison With Experimental Data," Journal of Engineering for Gas Turbines and Power, vol. 123, no. 4, p. 919, 2001.

[11] E. P. Petrov, "Explicit Finite Element Models of Friction Dampers in Forced Response Analysis of Bladed Disks," Journal of Engineering for Gas Turbines and Power, vol. 130, no. 2, p. 022502, 2008.

[12] M. Hajžman, L. Pešek, J. Brůha, V. Zeman, and D. Rychecký, "Basic optimization methodology for the design of friction damping in blade shrouds," in Proceedings of ASME 2013 International Design Engineering Technical Conferences and Computers and Information in Engineering Conference, vol. 7A, 2013.

[13] L. Pešek and L. Pust, "Influence of dry friction damping on bladed disk vibration," in Vibration Problems ICOVP 2011: The 10th International Conference on Vibration Problems, pp. 557-564, Springer Netherlands, 2011.

[14] J. H. Griffin, “An Integrated Approach for Friction Damper Design," Journal of Vibration and Acoustics, vol. 1, no. April 1990, pp. 175-181, 1990.

[15] M. H. Jareland, "A parametric study of a cottage-roof damper and comparison with experimental results," in Proceedings of ASME TURBOEXPO, pp. 1-9, 2001. 
[16] L. Panning, W. Sextro, and K. Popp, “Optimization of interblade friction damper design," in Proceedings of ASME TURBOEXPO, pp. 1-8, 2000.

[17] C. M. Firrone, "Measurement of the kinematics of two underplatform dampers with different geometry and comparison with numerical simulation," Journal of Sound and Vibration, vol. 323, pp. 313-333, June 2009.

[18] I. a. Sever, E. P. Petrov, and D. J. Ewins, "Experimental and Numerical Investigation of Rotating Bladed Disk Forced Response Using Underplatform Friction Dampers," Journal of Engineering for Gas Turbines and Power, vol. 130, no. 4, p. 042503, 2008.

[19] T. Berruti, "A test rig for the investigation of the dynamic response of a bladed disk with underplatform dampers," Mechanics Research Communications, vol. 37, pp. 581-583, Sept. 2010.

[20] T. Berruti, C. M. Firrone, and M. M. Gola, “A Test Rig for Noncontact Traveling Wave Excitation of a Bladed Disk With Underplatform Dampers," Journal of Engineering for Gas Turbines and Power, vol. 133, no. 3, p. 032502, 2011.

[21] E. P. Petrov and D. J. Ewins, "Advanced Modeling of Underplatform Friction Dampers for Analysis of Bladed Disk Vibration,” Journal of Turbomachinery, vol. 129, no. 1, p. 143, 2007.

[22] R. K. Giridhar, P. V. Ramaiah, G. Krishnaiah, and S. G. Barad, "Gas Turbine Blade Damper Optimization Methodology," Advances in Acoustics and Vibration, vol. 2012, pp. 1-13, 2012.

[23] C. W. Schwingshackl, C. Joannin, L. Pesaresi, J. S. Green, and N. Hoffmann, "Test method development for nonlinear damping extraction of dovetail joints," in Proceedings of the Society for Experimental Mechanics IMAC Conference, 2014.

[24] S. Smith, J. Bilbao-Ludena, S. Catalfamo, M. Brake, P. Reuß, and C. W. Schwingshack1, "The effects of boundary conditions, measurement techniques, and excitation type on measurements of the properties of mechanical joints," in Proceedings of the Society for Experimental Mechanics IMAC Conference, 2016.

[25] E. P. Petrov and D. J. Ewins, "State-of-the-art dynamic analysis for non-linear gas turbine structures," Proceedings of the Institution of Mechanical Engineers, Part G: Journal of Aerospace Engineering, vol. 218, pp. 199-211, Jan. 2004.

[26] E. P. Petrov and D. J. Ewins, "Analytical Formulation of Friction Interface Elements for Analysis of Nonlinear Multi-Harmonic Vibrations of Bladed Disks," Journal of Turbomachinery, vol. 125, no. 2, p. 364, 2003.

[27] E. P. Petrov, "A High-Accuracy Model Reduction for Analysis of Nonlinear Vibrations in Structures With Contact Interfaces," Journal of Engineering for Gas Turbines and Power, vol. 133, no. 10, p. 102503, 2011.

[28] C. W. Schwingshackl, E. P. Petrov, and D. J. Ewins, "Effects of Contact Interface Parameters on Vibration of Turbine Bladed Disks With Underplatform Dampers," Journal of Engineering for Gas Turbines and Power, vol. 134, no. 3, p. 032507, 2012. 
[29] C. W. Schwingshackl, E. P. Petrov, and D. J. Ewins, "Measured and estimated friction interface parameters in a nonlinear dynamic analysis," Mechanical Systems and Signal Processing, vol. 28, pp. 574-584, Apr. 2012.

[30] C. W. Schwingshackl, "Measurement of Friction Contact Parameters for Nonlinear Dynamic Analysis," in Proceedings of the Society for Experimental Mechanics IMAC Conference, 2012.

[31] J. S. Green, Controlling Forced Response of a High Pressure Turbine Blade. PhD thesis, KTH Royal Institute of Technology, 2006. 